\title{
Radiation damage studies in cardiac muscle cells and tissue using microfocused $X$-ray beams: experiment and simulation
}

\author{
Jan-David Nicolas, Sebastian Aeffner and Tim Salditt
}

J. Synchrotron Rad. (2019). 26, 980-990

\footnotetext{
IIUCr Journals CRYSTALLOGRAPHY JOURNALS ONLINE

Copyright (C) International Union of Crystallography

Author(s) of this article may load this reprint on their own web site or institutional repository provided that this cover page is retained. Republication of this article or its storage in electronic databases other than as specified above is not permitted without prior permission in writing from the IUCr.

For further information see http://journals.iucr.org/services/authorrights.html
} 


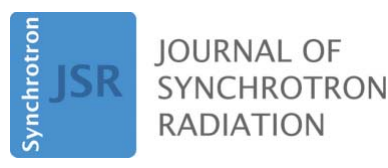

ISSN 1600-5775

Received 1 February 2019

Accepted 12 May 2019

Edited by E. F. Garman, University of Oxford, England

Keywords: radiation damage; scanning X-ray diffraction; microdiffraction; Monte Carlo simulation; EGSnrc.

Supporting information: this article has supporting information at journals.iucr.org/s

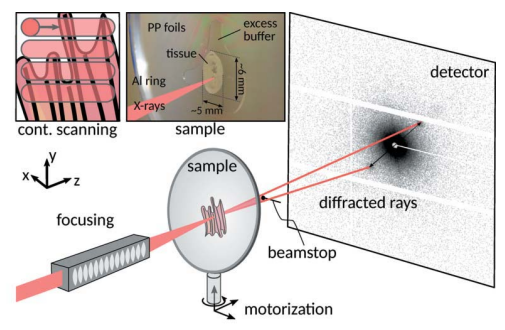

C 2019 International Union of Crystallography

\section{Radiation damage studies in cardiac muscle cells and tissue using microfocused X-ray beams: experiment and simulation}

\author{
Jan-David Nicolas, Sebastian Aeffner and Tim Salditt*
}

Georg-August-Universität Göttingen, Institut für Röntgenphysik, Friedrich-Hund-Platz 1, 37077 Göttingen, Germany. *Correspondence e-mail: tsaldit@gwdg.de

Soft materials are easily affected by radiation damage from intense, focused synchrotron beams, often limiting the use of scanning diffraction experiments to radiation-resistant samples. To minimize radiation damage in experiments on soft tissue and thus to improve data quality, radiation damage needs to be studied as a function of the experimental parameters. Here, the impact of radiation damage in scanning X-ray diffraction experiments on hydrated cardiac muscle cells and tissue is investigated. It is shown how the small-angle diffraction signal is affected by radiation damage upon variation of scan parameters and dose. The experimental study was complemented by simulations of dose distributions for microfocused X-ray beams in soft muscle tissue. As a simulation tool, the Monte Carlo software package EGSnrc was used that is widely used in radiation dosimetry research. Simulations also give additional guidance for a more careful planning of dose distribution in tissue.

\section{Introduction}

Scanning diffraction and in particular spatially resolved smallangle X-ray scattering (also denoted as scanning SAXS or sSAXS) is of interest for biological imaging and structure analysis, since it helps to probe biomolecular assemblies in their native environment rather than in idealized and homogeneous model systems. sSAXS was first used for biomaterials such as wood (Lichtenegger et al., 1999; Müller, 2009; Storm et al., 2015), bone (Wagermaier et al., 2007; Rinnerthaler et al., 1999; Gourrier et al., 2010; Karunaratne et al., 2012; Granke et al., 2013; Giannini et al., 2014; Bukreeva et al., 2015) and teeth (Tesch et al., 2001; Märten et al., 2010; Deyhle et al., 2011, 2014) as well as for soft tissues (Bunk et al., 2009; Müller et al., 2010; Giannini et al., 2019), with typical resolution values in the range of several micrometres. More recently, the spatial resolution has been significantly increased and the technique has been extended to biological cells, including freeze-dried (Weinhausen et al., 2012; Bernhardt et al., 2016, 2017), chemically fixed (Weinhausen et al., 2014) and even living cells (Weinhausen et al., 2014; Nicolas et al., 2019), as reviewed by Hémonnot \& Köster (2017).

Progress in X-ray optics and focusing together with improved source characteristics has made it possible to probe the structure of biological matter by diffraction in volumes down to the $(100 \mathrm{~nm})^{3}$ range, based on well controlled X-ray spot sizes. Elliptic mirrors in the Kirkpatrick-Baez geometry (KB mirrors), compound refractive lenses and Fresnel zone plates even achieve spot sizes below $100 \mathrm{~nm}$ (Sakdinawat \& Attwood, 2010; Stangl et al., 2014). By recording and evaluating millions of diffraction patterns collected over a raster- 
scanned sample, high resolution in reciprocal space can be combined with a spatial resolution (in real space) given by the beam or step size. In this way, spatially inhomogeneous and hierarchical samples become amenable for structure analysis by diffraction.

sSAXS is particularly well suited to elucidate multi-scale structures of biological systems, from the molecular and cellular up to the tissue and organ level. Heart contractility is one prominent example for a function which relies on such a multi-scale and multi-level structure. Important parameters, such as the orientation of muscle fibrils or the interfilament distance between myosin filaments in muscle fibrils, cannot be suitably assessed by conventional histology. sSAXS can fill this gap and can be used to assess how different regions within the heart exhibit characteristic structural variations. For example, the acto-myosin lattice spacing in the endocardium was found to differ from the myocardium (Nicolas et al., 2017a). Such experiments combine real space information with molecular sensitivity by diffraction, and hereby complement advanced optical methods which non-invasively visualize muscle motion in vivo and in vitro. A particular advantage of sSAXS is the large penetration depth which probes deeper layers rather than just the surface.

In this work, we address the detrimental influence of beam damage on structures probed by sSAXS, which is an important limitation when applying the technique to soft biological tissues. To this end, we study how the SAXS signal of a specific super-molecular structure decreases with increasing dose and different scan parameters. Specifically, we quantify changes in the equatorial peak of heart muscle tissue. The intensity of the reflection from the acto-myosin lattice provides a clear indication of structural damage. The equatorial reflections originate from the arrangement of the actin and myosin filaments in a hexagonal lattice in the sarcomere of muscle cells. Typically, the $(1,0)$ and $(1,1)$ equatorial reflections can be detected from this lattice with an approximate filament spacing of $45 \mathrm{~nm}$. For a detailed discussion of this signal and the corresponding structure, its spatial variation in heart tissue, as well as the prerequisites of sample preparation, instrumentation and data analysis, we refer the reader to Nicolas et al. (2019).

We complement the experimental results by Monte Carlo (MC) simulations of the spatial dose profile, which can guide the experimenter in the design of sSAXS experiments. The conclusions, drawn both from experimental results and supported by the MC simulations, are applicable to many other biological samples with radiation-sensitive structures. Further, the results carry over to coherent diffractive imaging with tightly focused beams, as in ptychography (Faulkner \& Rodenburg, 2004; Thibault et al., 2008). However, as we will see below, overlaps between exposed regions are particularly problematic.

Radiation damage to biological tissue is already a well studied subject, as reviewed for example by Hall \& Giaccia (2012). In the present context, however, we take an approach similar to Riekel et al. (2010) and Storm et al. (2015) that is specific with regard to tight focusing, scanning and the range of spatial frequencies probed, which is much smaller than in crystallography for example. The hypothesis is that, for sSAXS, one can find measurement conditions where relatively large spatial structures remain intact before enough chemical bonds have been broken during the time span of the acquisition. In order to ensure that the recorded signal is unaffected by radiation damage, scan parameters have to be varied and MC simulations must be carried out to judge the transport of dose by secondary processes, in particular by photoelectrons. Furthermore, radiation-created free electrons can also be transported by diffusion in hydrated tissue, as discussed by Hémonnot \& Köster (2017).

This work therefore integrates well with a vast body of research on radiation damage in X-ray crystallography (Garman \& Weik, 2017) where MC methods are, for example, being used to simulate dose distributions in small crystals (Nave \& Hill, 2005; Cowan \& Nave, 2008; Dickerson \& Garman, 2019), scan parameters and data collection methods being optimized (Zeldin et al., 2013; Owen et al., 2014; Gao et al., 2018), as well as smaller beams being employed (Sanishvili et al., 2011) to minimize radiation damage that would otherwise render crystallographic phasing impossible.

Before we present the experimental results and finally the MC simulations which support this view, we review the fundamental dosimetry definitions for notational clarity in the next section. A brief conclusion and further discussion of this study is given at the end of the manuscript.

\section{Dosimetry definitions and notation}

In general, calculating the absorbed dose distribution $D(\mathbf{r})$ for an arbitrary radiation field is a very complex task, especially in a non-homogeneous absorbing medium such as the human body [see Attix (2004) and McDermott (2016) for an overview]. To solve the complex problem of the energy transfer from microfocused X-ray beams to thin tissue sections, we use MC simulations that make use of the fundamental interactions between particles and matter and which can be used to reproduce physical reality with remarkable accuracy. Before describing the MC simulations in detail, basic definitions of radiation dosimetry are repeated for the purpose of notational clarity.

We write Beer-Lambert's law as

$$
\Phi=\Phi_{0} \exp (-\mu z)
$$

where $\Phi_{0}$ denotes the initial photon fluence (number of photons per unit area) of a parallel monochromatic X-ray beam, $\mu$ is the linear attenuation coefficient and $z$ is the coordinate in the beam direction. For hard X-rays, the linear attenuation coefficient $\mu=\mu(E)$ is a steep function of photon energy, scaling approximately as $\mu(E) \propto E^{-3}$, away from absorption edges, but to keep the notation simple the dependence on $E$ is not explicitly included.

After a secondary electron has been emitted (either by the photoelectric effect or by the Compton effect), it undergoes multiple collisions with atomic electrons of the surrounding tissue (causing excitations and ionizations) and loses its kinetic energy in a quasi-continuous fashion, until it ultimately comes 
to rest. These electron-electron collisions (and not the primary photon interactions) are the central mechanism by which photon energy is converted into an absorbed dose. The finite mean distance of the electron before it is fully absorbed by the medium is termed the electron range $R$. The two relevant and material-dependent length scales for dosimetry of photon beams are therefore the photon attenuation length $\mu^{-1}$ and the electron range $R$.

For photon energies considered here, the mass attenuation coefficient $\mu / \rho$ can be written as

$$
\frac{\mu}{\rho}=\frac{N_{\mathrm{A}}}{A}\left(\sigma_{a}^{\mathrm{p} . \mathrm{e} .}+\sigma_{a}^{\mathrm{Th}}+\sigma_{a}^{\mathrm{C}}\right),
$$

where $\sigma_{a}^{\text {p.e. }}, \sigma_{a}^{\text {Th }}, \sigma_{a}^{\text {C }}$ denote the atomic cross-sections for the photoelectric effect, Thomson scattering and Compton scattering, respectively, $\rho$ is the density of the material, $A$ is the molar mass of the material and $N_{\mathrm{A}}$ is Avogadro's constant. A simple analytical dose calculation is only possible under the conditions of either radiation equilibrium or the less restrictive case of charged particle equilibrium (CPE). The conditions for $\mathrm{CPE}$ are (approximately) realized within a broad, parallel and homogeneous X-ray beam with a diameter considerably larger than twice the electron range $R$, such that edge effects can be neglected in the beam center. In this case, the dose can be estimated from

$$
D=\left(\frac{\mu_{\mathrm{en}}}{\rho}\right) E \Phi,
$$

where $\mu_{\mathrm{en}} / \rho$ is the mass energy absorption coefficient [which under the described conditions is very close to the linear attenuation coefficient $\mu$ (see Attix, 2004)], $E$ is the photon energy and $\Phi$ is the photon fluence of a monoenergetic photon field. The photon fluence can be expressed as $\Phi=I_{0} \tau / \Delta_{x} \Delta_{y}$, where $\tau$ is the exposure time, $I_{0}$ is the beam intensity and $\Delta_{x} \Delta_{y}$ is the size of the X-ray beam. In fact, equation (3) can be used to independently verify the quality of the MC simulations with EGSnrc (Kawrakow, 2000), the MC simulation tool used in Section 4. For the purpose of validation, a $40 \mu \mathrm{m}$ broad, uniform parallel beam was simulated and the dose was calculated in the center of the illuminated area at a depth where the CPE approximation is valid. The dose obtained was in excellent agreement with the dose estimated from equation (3). Since the conditions for CPE include a uniform photon field, equation (3) can, strictly speaking, not be applied to the case of microfocus X-ray beams, where $\Phi$ varies from its maximum value close to zero on length scales of the order of $1 \mu \mathrm{m}$. However, since $z \ll \mu^{-1}$ is usually fulfilled for tissue sections with a thickness below $100 \mu \mathrm{m}, \mathrm{CPE}$ does exist along the longitudinal coordinate $z$, as will be seen in Section 4 .

\section{Experiment}

Radiation damage recordings on cardiac tissue sections were obtained at the microbeam section of beamline ID13 of the European Synchrotron Radiation Facility (ESRF). A photon energy of $13.0 \mathrm{keV}$ was selected and a focused beam of approximately $\Delta_{x} \Delta_{y}=2.9 \mu \mathrm{m}$ (horizontal) $\times 1.4 \mu \mathrm{m}$ (vertical) (full width at half-maximum, FWHM) and a divergence of $0.30 \mathrm{mrad}$ was produced by selection of a suitable combination of compound refractive lenses within a transfocator. The beam divergence was estimated from a focal length of $164.5 \mathrm{~mm}$ and the $50 \mu \mathrm{m}$-diameter circular aperture of the focusing lenses, and using $2 \tan ^{-1}(25 \mu \mathrm{m} / 164.5 \mathrm{~mm})=0.30 \mathrm{mrad}$. The beam size was obtained from horizontal and vertical scanning of a gold wire through the focus of the X-ray beam, recording a line plot of the transmitted intensity with a calibrated silicon diode. The beam size was then determined from a Gaussian fit to the derivative of the curves. The diode was also used to calculate the total photon flux $I_{0}=1.57 \times 10^{12}$ photons s $^{-1}$. A two-dimensional single-photon-counting pixel detector (Eiger $4 \mathrm{M}$, Dectris) was placed $0.97 \mathrm{~m}$ behind the sample for fast diffraction data recording. The position of the primary beam on the detector, detector tilt and detector distance were calibrated using silver behenate as a reference standard material. The dose $D$ for cardiac tissue and an exposure time of $\tau=$ $10 \mathrm{~ms}$ was estimated from $D=\mu I_{0} \tau E / \rho \Delta_{x} \Delta_{y}=2.54 \mathrm{MGy}$ (Howells et al., 2009), with $\mu / \rho=3.1582 \mathrm{~cm}^{2} \mathrm{~g}^{-1}$ linearly interpolated for skeletal muscle at $13.0 \mathrm{keV}$ from the XAAMDI database and $\Delta_{x} \Delta_{y}$ the area probed by the beam.

Radiation damage studies were conducted on $30 \mu \mathrm{m}$-thick cardiac tissue sections. The heart tissue was obtained from a wildtype C57BL/6 mouse (female, age: 100 days). Following the excision, the heart was shortly washed with phosphatebuffered saline (PBS), then chemically fixed overnight using $10 \%$ formaldehyde solution at room temperature (RT). The sample was then embedded in agarose (5\% in PBS) at RT and directly sectioned using a vibratome (VT1000 S; Leica Biosystems, Germany). For sectioning, the sample was immersed in $4^{\circ} \mathrm{C}$ cold PBS. The tissue sections were then stored for 13 days at $4^{\circ} \mathrm{C}$ in PBS containing $0.04 \%$ sodium azide until the experiment. To prepare the sample for the experiment, the tissue sections were sandwiched between two polypropylene foils of approximately $1 \mu \mathrm{m}$ thickness with excess buffer added to the chamber to keep the sample moist during the experiment. The chamber was sealed using nail polish and mounted onto a motorized stage that can move the sample at constant speed along the $x$-axis [see Fig. 1(a)]. A stepping motor then moves the sample in discrete steps along the $y$-axis. Since the sample is now not sequentially stepped along the $x$-axis but moved at constant speed, the step size is defined in this work as the distance traveled between the start of two successive exposures. We have chosen this scanning mode as it eliminates any inertial effects and data acquisition overhead, ultimately making large scans on millimetre-sized tissue samples feasible.

The overall scattering geometry and an image of a mounted sample is shown in Fig. 1(A). It was previously demonstrated that such a diffraction recording scheme in combination with automated data treatment yields valuable structure information in every scan point such as lattice spacings, orientation and order parameters. Even millimetre-sized objects can now be raster scanned with reasonable recording times. As an example, a raster map showing the integrated intensity for a $30 \mu \mathrm{m}$-thick cardiac tissue section is shown in Fig. 1(B). 


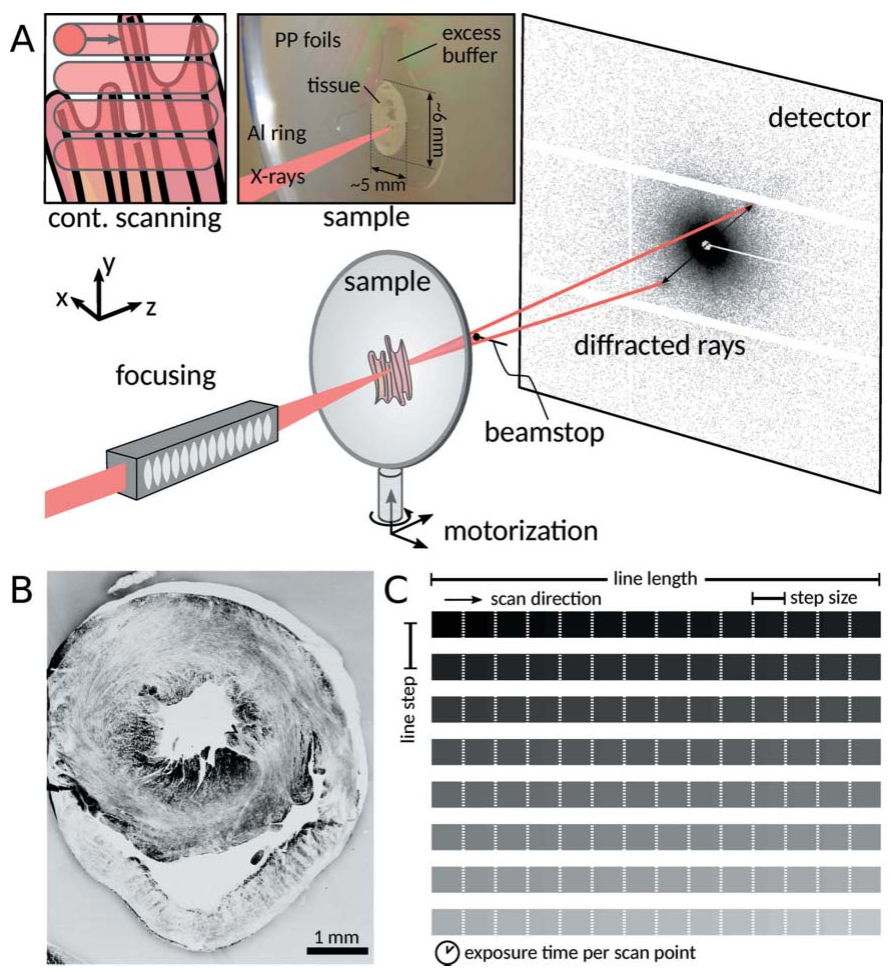

Figure 1

(A) Sketch of the experimental setup. A tissue section is scanned with a microfocused or nanofocused synchrotron beam. For microfocusing at beamline ID13, ESRF, a transfocator equipped with compound refractive lenses is commonly used. The tissue is sandwiched between two polypropylene foils and several microlitres of buffer solution are added to keep the sample moist during the experiment. It is swept continuously along the $x$-axis and stepped along the $y$-axis through the $\mathrm{X}$-ray focus, and diffraction patterns are collected at regular time intervals. A beamstop is used to block the primary (unscattered) beam. (B) Map of the integrated intensity of a tissue section described in detail by Nicolas et al. (2017a). (C) Scan parameters that can be varied to minimize the detrimental effect of radiation damage.

Regarding radiation damage, several scan parameters can be varied to minimize its detrimental effect on data quality. The scan parameters that can be easily tuned are the line length, step size, line step and the exposure time per scan point, as shown in Fig. 1(C).

We have therefore carried out an experiment in which we have varied all four parameters highlighted in Fig. 1(C) as well as the dose per point. To quantify the effect of radiation damage, we have monitored the intensity of the $(1,1)$ equatorial reflection, which can be prominently observed at $0.28 \mathrm{~nm}^{-1}$ in a background-subtracted and averaged diffraction pattern shown in Fig. 2(A), while the $(1,0)$ reflection is blocked by the beamstop. To extract the intensity of the $(1,1)$ reflection, all patterns from a scan were averaged, background was subtracted and data were azimuthally integrated. 1D profiles were then modeled using a power-law decay and a Gaussian, $I\left(q_{r}\right)=a q_{r}^{-b}+c \exp \left[-\left(q_{r}-q^{(1,1)}\right)^{2} / \sigma\right]$. The reflection position $q^{(1,1)}$ and the reflection width $\sigma$ were held constant at $0.28 \mathrm{~nm}^{-1}$ and $0.034 \mathrm{~nm}^{-1}$, respectively. As an example, seven 1D profiles in Fig. 2(B) show the decay of the reflection amplitude as a function of the line step parameter.

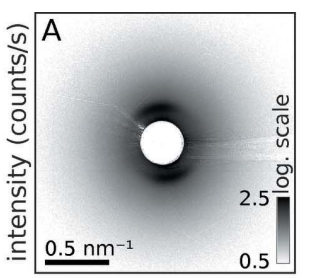

dose (MGy)
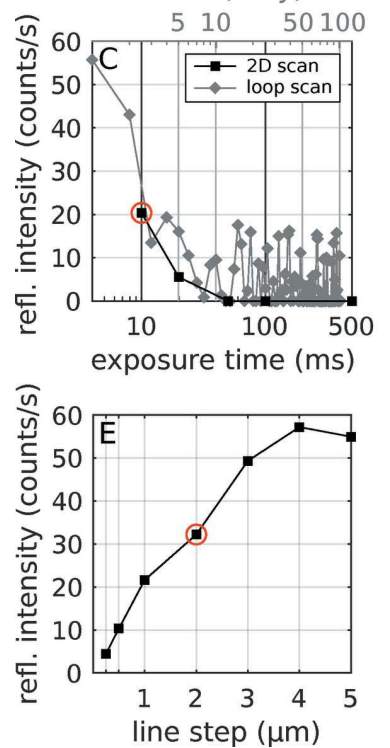

Figure 2

Change of the myosin (1,1) reflection intensity with varying scan parameters and increasing dose. (A) Average scattering pattern from a scan with $5 \mu \mathrm{m}$ line step, showing the $(1,1)$ myosin reflection at approximately $0.28 \mathrm{~nm}^{-1}$. (B) Variation of the line step parameter showing a decay of the azimuthally integrated profiles. Each profile is fitted using a simple power law decay and a Gaussian. (C)-(F) The extracted peak intensity is shown for a variation of four scan parameters. Black lines serve as guides to the eye. A red circle in each graph marks the parameter value that was used in scans where one of the three other parameters was varied. (C) The exposure time has also been varied without scanning, such that a single spot was repeatedly exposed (loop scan), as shown in gray. For these data, the accumulated dose is shown as a second axis. Upon variation of the exposure time, the peak intensity has already completely vanished at $50 \mathrm{~ms}$. (D) The variation of the length of a scan line has no effect on the reflection intensity. (E) The contrary can be seen for a variation of the line step. The peak intensity continuously increases until a plateau is reached at approximately $4 \mu \mathrm{m}$. (F) Increasing the step size also leads to an increase in reflection intensity.

In the following, the intensity of the $(1,1)$ reflection is reported as a function of scan parameters. Importantly, for each scan, the remaining scan parameters have to be kept constant and are marked by red circles in the respective graphs shown in Figs. 2(C)-2(F). For instance, all scans for which the exposure time was varied were recorded using a line length of $100 \mu \mathrm{m}$ and a line step and step size of $2 \mu \mathrm{m}$, as marked by red circles in Figs. 2(D)-2(F). In Fig. 2(C), a solid black line marks the decay of the reflection intensity with increasing exposure time. Exposure times of $\{10,20,50,100,500\}$ ms were sampled. A minimal exposure time of $10 \mathrm{~ms}$ was clearly optimal, since already $20 \mathrm{~ms}$ leads to a significant decay in the intensity of the $(1,1)$ reflection. In addition to scanning, the exposure time was also varied while keeping the beam spot on a single position 
in the tissue, which was continuously probed. In this case, a similar decay in the reflection intensity was observed, as marked by a solid gray line as a function of both time and dose in Fig. 2(C). Note that a linear relationship was assumed here for the case of negligible mass loss, which can become significant depending on the experimental parameters (Beetz \& Jacobsen, 2003), but which we could not quantify here.

The length of a scan line, $\{100,150,200,500,1000\} \mu \mathrm{m}$, shown in Fig. 2(D), does not impact the quality of the signal given a $10 \mathrm{~ms}$ exposure time and conservative values of $2 \mu \mathrm{m}$ for the line step and step size.

A strong effect on the reflection intensity can be observed when reducing the distance between two adjacent lines, see Fig. 2(E). Here, we have used spacings of $\{0.25,0.5,1,2,3$, $4,5\} \mu \mathrm{m}$. For a beam of vertical size $1.4 \mu \mathrm{m}$ (FWHM) one might expect that the reflection intensity decreases once the line step was reduced below approximately $2 \mu \mathrm{m}$ as one begins to have a considerable overlap between adjacent scan lines. However, a decrease was already observed when the line step was reduced to less than $4 \mu \mathrm{m}$, indicating that the range of photoelectrons is larger than the beam size in this experiment. A photoelectron range of $4 \mu \mathrm{m}$ has also been measured for protein crystals at $100 \mathrm{~K}$ and an energy of $18.5 \mathrm{keV}$ (Sanishvili et al., 2011). This result is also in excellent agreement with $\mathrm{MC}$ simulations as will be discussed in the following section. We also take this behavior as an indication that the observed radiation damage occurs due to direct ionization and free radicals formed through the ionization of water. Secondary processes can cause damage at more distant locations due to diffusion of more stable free radicals, but involve time scales associated, first, with the generation (build-up) of free radicals, second, their transport by diffusion and, third, the chemical reactions resulting in structural damage (Riley, 1994). For the experimentally relevant time scales (acquisition time, scanning times) and length scales, we do not have any indications of secondary damage affecting the diffraction signal. If the opposite were true, the reflection intensity would not have saturated at a line step of around $4 \mu \mathrm{m}$ but would have further increased, since a larger spacing between the lines should have reduced any effect of secondary damage. It is hence not further discussed.

Finally, the step size was set to $\{0.25,0.5,1,2\} \mu \mathrm{m}$ [Fig. 2(F)]. Here, we observed a pronounced decrease in reflection intensity upon decreasing the step size. In fact, the $(1,1)$ reflection is barely detectable anymore at a step size below $1 \mu \mathrm{m}$. We conclude that step sizes below $2 \mu \mathrm{m}$ are not reasonable, due to an increase in dose as the scanning speed is reduced for a constant exposure time.

The above description was based on scanning experiments on thin tissue sections. However, as was recently demonstrated, similar structural information can also be probed within single, isolated biological cells (Nicolas et al., 2019). In such experiments, it is less useful to perform in silico dose simulations since it is more questionable for a cell to be described by bulk material properties than for a tissue slice, which at least has a constant thickness. To obtain a rough estimate of the radiation sensitivity of the underlying struc-
Table 1

Experimental configuration of beamline P10 at the PETRA III storage ring, DESY.

Parameters of the scanning diffraction experiments on freeze-dried and isolated adult cardiomyocytes. For an estimation of the dose $D$ per point, a mass attenuation coefficient of $\mu / \rho=9.88 \mathrm{~cm}^{2} \mathrm{~g}^{-1}$ was used, following Howells et al. (2009).

\begin{tabular}{lll}
\hline Sample preparation & Freeze-dried & Hydrated \\
\hline Energy $(\mathrm{keV})$ & 7.5 & 13.8 \\
Monochromator & $\mathrm{Si}(111)$ channel-cut & $\mathrm{Si}(111)$ channel-cut \\
Detector & Eiger $4 \mathrm{M}$ & Eiger $4 \mathrm{M}$ \\
Detector distance $(\mathrm{m})$ & 5.413 & 5.045 \\
Focus $(\mu \mathrm{m})$ & $1.4 \times 2.4$ & $2.3 \times 2.3$ \\
Focusing & Compound refractive & Compound refractive \\
& $\quad$ lenses & lenses \\
$I_{0}($ photons s & -1 \\
Exposure time $\tau$ & $2.1 \times 10^{10}$ & $8.6 \times 10^{9}$ \\
Dose $D$ per point & $10 \mathrm{~ms}$ & $1000 \mathrm{~ms}$ \\
Dose rate & $74 \mathrm{kGy}^{-1}$ & $3.6 \mathrm{MGy}^{-1}$ \\
\hline
\end{tabular}

ture, successive exposures were used to progressively increase the absorbed energy and record changes in the scattering patterns. In SAXS experiments on single cells, two common sample preparation approaches have been used, depending on the flux density used in the experiment. From previous studies on adult cardiomyocytes we inferred that, when using KB

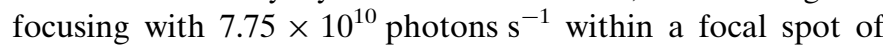
approximately $300 \mathrm{~nm} \times 300 \mathrm{~nm}(\mathrm{FWHM})$, freeze-drying is a well suited sample preparation method for cells at RT. However, if relaxed focusing of the order of $2 \mu \mathrm{m} \times 2 \mu \mathrm{m}$ (FWHM) with a photon flux of $8.6 \times 10^{9}$ photons s $^{-1}$ is used, cells could be chemically fixed and kept in solution during the experiment (Nicolas et al., 2019).

The following section is concerned with adult cardiomyocytes isolated from wild-type mouse hearts following the protocol described by Nicolas et al. (2019). Following isolation from the tissue, cells with a typical size of approximately $20 \mu \mathrm{m} \times 100 \mu \mathrm{m}$ were either chemically fixed and kept hydrated until and during the experiment or chemically fixed, plunge-frozen and subsequently freeze-dried. For both sample preparations, a series of scattering patterns was collected at the GINIX instrument of beamline P10, located at the PETRA III storage ring, DESY. All relevant experimental parameters for both experiments are summarized in Table 1.

In the case of freeze-dried preparations, scattering patterns of the form shown in Fig. 3(A) can be observed. One notable difference to those from hydrated tissue is the absence of equatorial reflections due to the invasive sample preparation. The one-dimensional structure factor shown in Fig. 3(B) decays with increasing dose. Each graph can also be normalized to the intensity of the first graph $I(D=74 \mathrm{kGy})$. In such a way, the $q$-dependent intensity loss can be seen more clearly, as shown in Fig. 3(C). Due to the absence of an equatorial reflection, each curve was now modeled by a simple power-law decay of the form $a q_{r}^{b}+c$. The power-law decay constant $b$ is shown in Fig. 3(D) as a function of dose $D$.

The hydrated specimens were not intensely processed prior to data collection and the highly ordered arrangement of myosin filaments remains intact, although the overall signal 

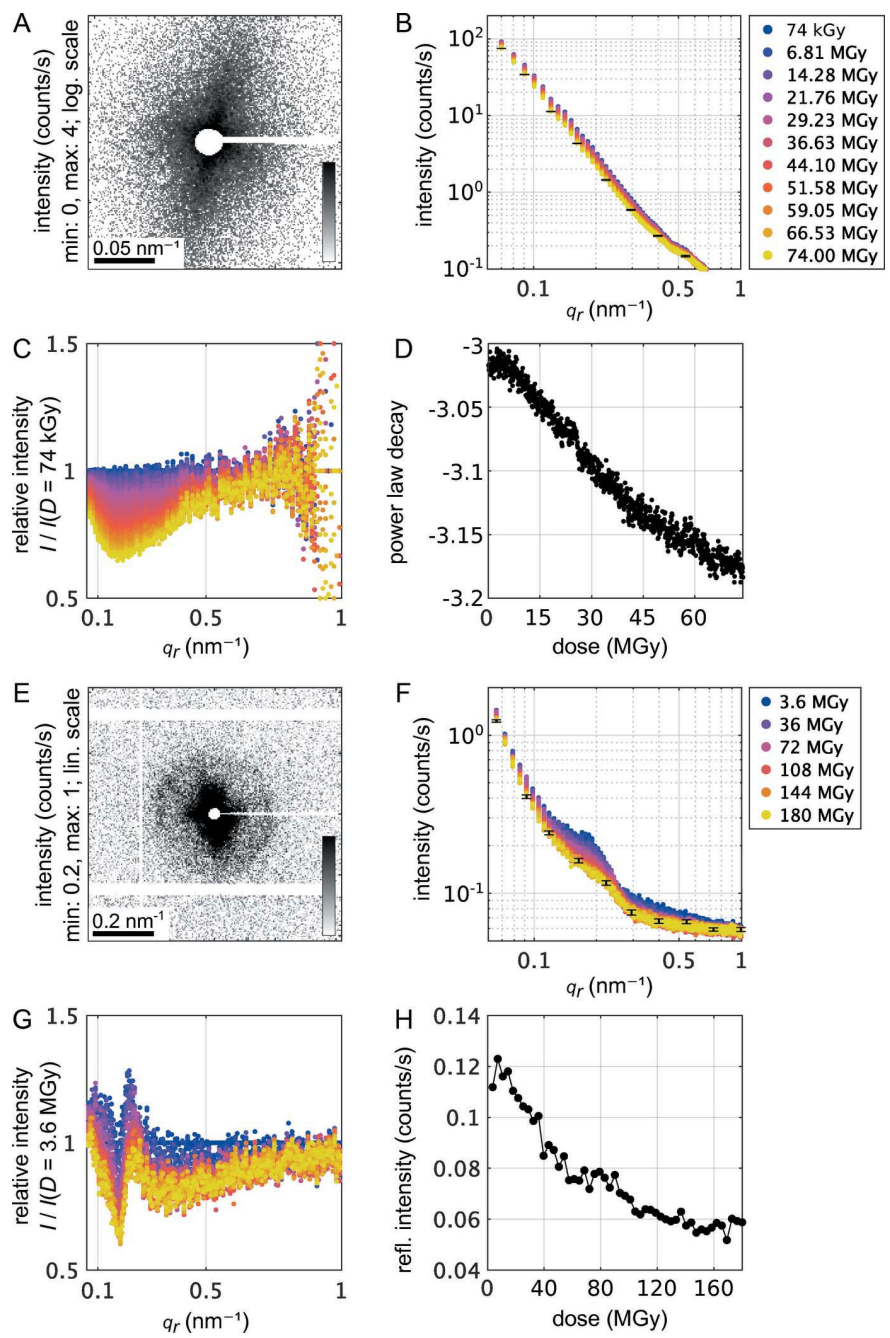

Figure 3

(A) Scattering signal of a freeze-dried adult cardiomyocyte. (B) Decay of the azimuthally integrated scattering intensity upon repeated illumination of an isolated location in the cell. Error bars are shown for $D=$ 74 MGy. (C) Normalization of all graphs to the intensity of the first exposure clearly shows the $q$-dependent intensity loss. (D) Power-law decay constant obtained from modeling the structure factor by a simple power law. (E) Scattering pattern from a hydrated, chemically fixed, adult cardiomyocyte. The $(1,0)$ equatorial reflection can be weakly observed in the scattering pattern using a microfocused beam of size $2.3 \mu \mathrm{m} \times 2.3 \mu \mathrm{m}$. (F) Repeated exposure of a fixed spot on the sample produces a resolution-dependent decay of the scattering signal, shown together with representative error bars for $D=180 \mathrm{MGy}$. (G) Inspection of the relative intensity reveals, that the $(1,0)$ reflection decays faster than the overall small-angle scattering signal. $(\mathrm{H})$ Intensity decay of the $(1,0)$ equatorial reflection.

level is greatly reduced compared with tissue sections. In an azimuthal integration of the two-dimensional scattering patterns shown in Fig. 3(E), the $(1,0)$ equatorial reflection gives rise to a Gaussian peak in the one-dimensional structure factor as shown in Fig. 3(F). The fast decay of the lattice reflection can be more readily observed in Fig. $3(\mathrm{G})$ where the relative intensity is plotted as a function of dose. The strongest decay in intensity can be observed at the reciprocal coordinate of the lattice reflection. As before, data were normalized to the first curve of the dataset. Once again, as for the case of cardiac tissue, the intensity decay was modeled using a power- law decay and a Gaussian, although in this case only the reflection width was held constant at $0.034 \mathrm{~nm}^{-1}$. The position of the reflection slightly shifts to higher values in $q_{r}$. The reflection intensity can now be plotted as a function of dose, as shown in Fig. 3(H). For both preparations, hydrated and freeze-dried, the dose was increased well beyond the Henderson limit of $20 \mathrm{MGy}$ (Henderson, 1990). In contrast to data shown in Fig. 3(D), in Fig. 3(H) one can see a leveling off of the reflection intensity beyond the Henderson limit.

The data show that the muscle filament structure can even tolerate doses in the MGy range, at RT and at a resolution given by the $(1,0)$ reflection. If even finer features of the sarcomeric structure such as meridional reflections are measured, as has been commonly carried out in X-ray diffraction for several decades (Matsubara, 1980), the effect of radiation damage on such reflections needs to be carefully reconsidered.

\section{Monte Carlo simulation}

The MC method is considered the gold standard for dose calculation in radiation dosimetry. It is based on the simulation of a very large number of particle histories, i.e. the track of an incident particle and all subsequent interactions with the material. An in-depth treatment of the MC method has been given by Seco \& Verhaegen (2013).

Tools such as the simulation suite CASINO (Drouin et al., 2007) have already been used in synchrotron radiation research, for example in simulating photoelectron escape from small protein crystals (Nave \& Hill, 2005; Cowan \& Nave, 2008). Here, we aim at using a suitable MC simulation scheme for the special case of scanning X-ray diffraction with microfocused synchrotron beams. For this purpose, MC simulations were carried out using the EGSnrc code system [GUI egs_inprz and user code DOSRZnrc for dose calculation in cylindrical geometries, see https://www.nrc-cnrc.gc.ca/eng/ solutions/advisory/egsnrc_index.html as well as Kawrakow (2000) and references therein]. As an input to the simulation we have made the following assumptions. First, the X-ray beam is assumed to be parallel and monochromatic with typical photon energies around $10 \mathrm{keV}$. The particular choice of energy is not crucial due to the absence of absorption edges in this energy range and for the four-component soft tissue standard concerned with here. The beam shape can be approximated as a Gaussian profile $P(r)=$ $\left(2 \pi \sigma^{2}\right)^{-1 / 2} \exp \left(-r^{2} / 2 \sigma^{2}\right)$, which is a reasonable approximation to the beam profile in the waist of a microfocused beam. It follows that the FWHM is given by the relation FWHM = $2(2 \ln 2)^{1 / 2} \sigma$, where $\sigma$ is defined as the $1 / e^{2}$ width of the beam. Secondly, the beam is perpendicularly incident from a vacuum region onto a perfectly flat and homogeneous layer of material. Thirdly, as a generic reference material representative for soft biological tissues, the ICRU four-component soft tissue (NIST standard material $262 ; \mu / \rho=4.937 \mathrm{~cm}^{2} \mathrm{~g}^{-1}$ and $R=2.538 \mu \mathrm{m}$ for $E=10 \mathrm{keV}$ ) was used, if not stated otherwise. The four-component tissue material composition is summarized in Table 2. 
To obtain a general feeling for dose distributions in a soft tissue matrix with a microfocused X-ray beam, the dose distribution was simulated for a beam with a FWHM of $1 \mu \mathrm{m}$. The radially symmetric dose profile is shown as a function of the radial $(r)$ and depth $(z)$ coordinate in Fig. 4. Using a logarithmic scaling along $z$, all relevant phenomena can be shown in a single figure and it can be more clearly seen that the dose distribution can be separated into three principal regions. In the dose buildup region where $z<R$, the dose increases and spreads with depth until it reaches an equilibrium region, the longitudinal charged particle equilibrium (CPE), where the overall transverse dose profile does not change as long as absorption does not play a significant role, i.e. $z \ll \mu^{-1}$. In this depth region $R<z \ll \mu^{-1}$, the radial dose profile is therefore essentially independent of $z$. A halfvalue layer can be defined at $z=(\ln 2) / \mu$ where the dose profile has been reduced to half of its maximal value until it finally drops (close to) zero for $z=\mathcal{O}\left(\mu^{-1}\right)$. Lastly, it is important to keep in mind that Fig. 4 and the following figures represent the dose per incident photon as an average quantity over a very large number of randomly sampled events.

Based on Fig. 4 the results from the previous theoretical considerations are well reproduced in that both length scales relevant in dose calculation and measurement (dosimetry) of photon beams, the photon attenuation length $\mu^{-1}$ and the electron range $R$ can be clearly distinguished. The results presented in Fig. 4 can be easily reproduced by the reader based on the documentation described in Appendix $A$.

In a visualization of the single tracks of one-hundred $10 \mathrm{keV}$ photons [green in Fig. 5(A)] that are constrained to interact in a $10 \mu \mathrm{m}$-thick tissue section to generate secondary electrons [blue in Fig. 5(A)], one can further observe that most photoelectrons are emitted roughly perpendicular to the direction of the primary photons. This is due to the nature of the photoelectric effect that occurs predominantly for $K$-shell electrons. The corresponding differential cross-section is (Leroy \& Rancoita, 2013)

$$
\left(\frac{\mathrm{d} \sigma}{\mathrm{d} \Omega}\right)_{a}^{\text {p.e., K }} \propto Z^{5} \mathcal{E}^{-7 / 2} \frac{\sin ^{2} \theta \cos ^{2} \phi}{(1-\beta \cos \theta)^{4}}
$$

for $\mathcal{E}:=h v / m_{\mathrm{e}} c^{2} \ll 1$, where $h v$ is the photon energy and $m_{\mathrm{e}} c^{2}$ is the rest energy of an electron, and $\beta:=v / c \ll 1$ with the particle velocity $v . \theta$ denotes the scattering angle with respect to the direction of the incident photon beam and $\phi$ the angle between the scattering and polarization planes. The maximum of photoelectron emission occurs for $\theta$ close to $90^{\circ}$ (with a slight tilt into the forward direction at $E=10 \mathrm{keV}$ ) and $\phi=$ $0^{\circ}$. Unfortunately, the $\phi$-dependence of the scattering due to polarization of the incoming photon beam is not yet included in MC dose calculations using EGSnrc.

Figure 5
Table 2

Four-component soft tissue.

\begin{tabular}{lll}
\hline Element & $Z$ & $\begin{array}{l}\text { Mass fraction } \\
\text { in percent }\end{array}$ \\
\hline $\mathrm{H}$ & 1 & 10.12 \\
$\mathrm{C}$ & 6 & 11.10 \\
$\mathrm{~N}$ & 7 & 2.60 \\
$\mathrm{O}$ & 8 & 76.18 \\
\hline
\end{tabular}

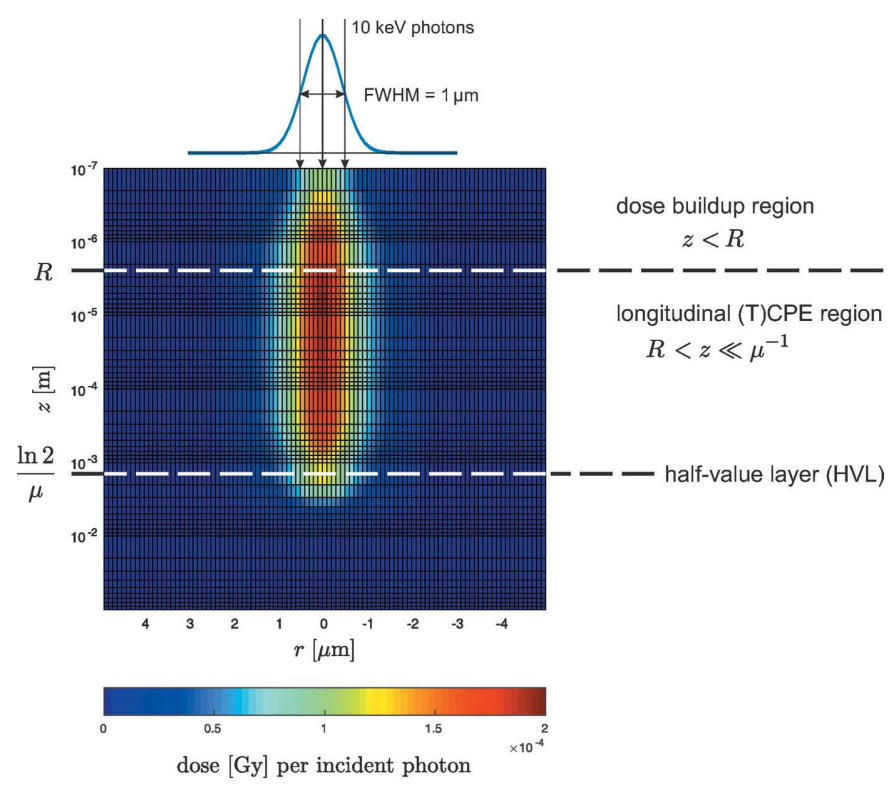

Figure 4

Dose distribution of a microfocus X-ray beam in soft tissue simulated with the EGSnrc MC code. In order to show all the relevant phenomena in a single figure, the $z$-axis in the direction of the incident photons is plotted on a logarithmic scale. In the beam direction, the dose distribution is determined by the buildup effect for $z<R$, remains approximately constant for $R<z \ll \mu^{-1}$ and finally drops to (close to) zero for $z=$ $\mathcal{O}\left(\mu^{-1}\right)$. In the radial direction, the extent of the region receiving relevant dose is determined by the photoelectron range $R$. Due to the generation of secondary photons (Thomson scattering, fluorescence, bremsstrahlung), there is some dose for $r>R$.
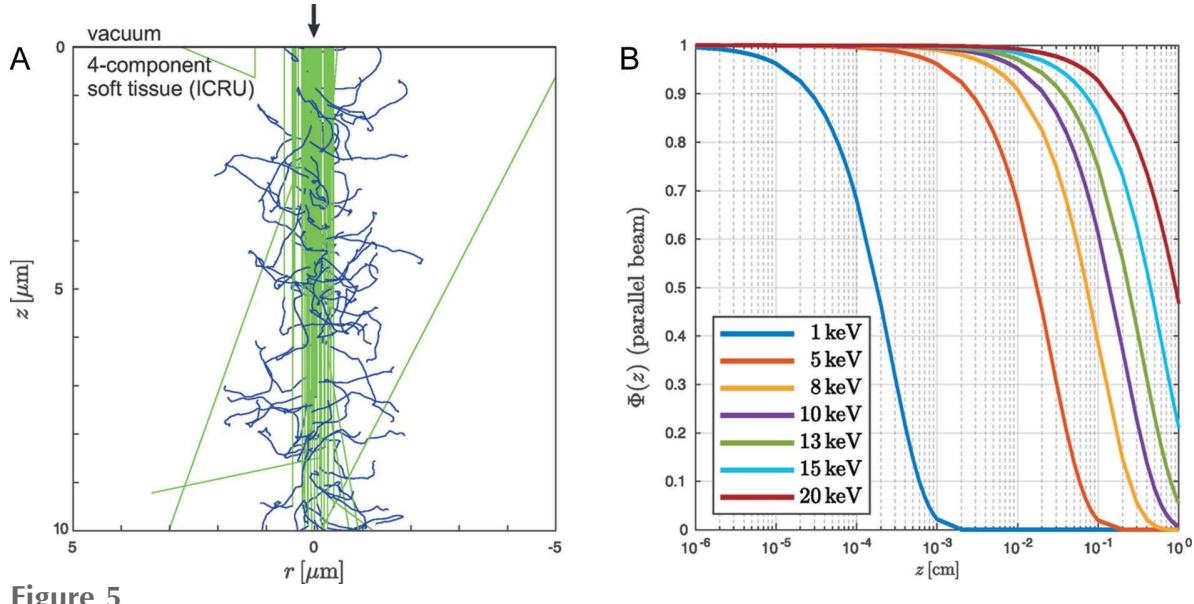

(A) MC simulation of one-hundred $10 \mathrm{keV}$ photons of a parallel beam (unpolarized, FWHM = $1 \mu \mathrm{m}$ ) which are constrained to interact in a $10 \mu \mathrm{m}$-thick layer of soft tissue (EGSnrc: option photon forcing enabled). Photon tracks are shown in green, electron tracks in blue. (B) Photon attenuation of photons in soft tissue according to the Beer-Lambert law $\Phi(z)=\Phi_{0} \exp [-\mu(E) z]$ plotted for different photon energies $E$ on a semi-logarithmic scale. For energies above $10 \mathrm{keV}$, attenuation can be neglected if the sample thickness is less than about $100 \mu \mathrm{m}$. 
The attenuation of $\mathrm{X}$-ray beams, which follows the BeerLambert law $\Phi(z)=\Phi_{0} \exp [-\mu(E) z]$, is depicted in Fig. 5(B) on a semi-logarithmic scale for common energies in microfocus experiments. As can be seen, for energies above $10 \mathrm{keV}$, as used in the actual experiments reported above, attenuation can be neglected if the sample thickness is less than about $100 \mu \mathrm{m}$, which is clearly fulfilled for the thin cardiac tissue sections described here.

EGSnrc can also be used to simulate pencil-beam dose kernels, shown here for six relevant X-ray energies $E=\{5,10$, $13,15,20,25\} \mathrm{keV}$. A pencil beam is a beam that approximates a delta-peak shape. In the simulation a parallel beam was simulated with a width of $50 \mathrm{~nm}$. Three two-dimensional dose profiles for $5 \mathrm{keV}, 10 \mathrm{keV}$ and $15 \mathrm{keV}$ are shown in Fig. 6. The beam footprint, here with a minimal width of a single resolution element, can be clearly distinguished from the overall dose profile. On a logarithmic scale the dose profile appears to have a sharp cutoff at approximately $R$. The kinetic energy of photoelectrons increases with primary X-ray photon energy, and, for $15 \mathrm{keV}$, photoelectrons can therefore penetrate deeper into the tissue. One can further observe a 'noise plateau' for $r>R$ which can be attributed to the re-emission of photons from secondary charged particles in the form of bremsstrahlung, fluorescence and annihilation radiation. This coupled transport of photons and electrons leads to a dose background that is almost independent of the sample dimensions.

Pencil-beam dose kernels not only show the dose distribution for beam sizes of minimal dimensions but can also be used to calculate dose profiles for extended beams by convolution. The so-called pencil-beam convolution method is used extensively in so-called convolution/superposition algorithms for external radiation therapy by medical linear accelerators with photon energies of several $\mathrm{MeV}$. However, the same principles can easily be applied to the case of microfocus $\mathrm{X}$-ray beams in the $\mathrm{keV}$ range. In this formalism dose kernels need to be calculated only once for each combination of photon energy and material. Subsequently, they can be applied to arbitrary beam shapes. In radiation therapy, further
Table 3

Glass support.

\begin{tabular}{llc}
\hline Element & $Z$ & $\begin{array}{l}\text { Mass fraction } \\
\text { in percent }\end{array}$ \\
\hline $\mathrm{O}$ & 8 & 45.98 \\
$\mathrm{Na}$ & 11 & 9.64 \\
$\mathrm{Si}$ & 14 & 33.66 \\
$\mathrm{Ca}$ & 20 & 10.72 \\
\hline
\end{tabular}

modifications are required to take into account beam divergence, continuous photon energy spectra and the irregular shape and inhomogeneities of the human body. For the present case of microfocus X-ray beams and thin homogeneous samples, no further modifications are required.

One final aspect of dose in thin tissue sections that is also of experimental interest is dose enhancement due to a substrate. If a tissue sample is placed on a substrate with higher mass density and composed of atoms with higher atomic number $Z$, this results in an increased dose due to back-scattered secondary electrons $\left(\theta>90^{\circ}\right)$ in the vicinity of the tissue/ substrate interface. As an example, the following geometry is considered (compare Fig. 7): (i) parallel, Gaussian beam with $1 \mu \mathrm{m}$ FWHM, as above, and (ii) $5 \mu \mathrm{m}$ layer of four-component soft tissue on top of a $5 \mu \mathrm{m}$-thick glass substrate with a mass density of $2.4 \mathrm{~g} \mathrm{~cm}^{-3}, \mu=61.58 \mathrm{~cm}^{-1}$ and $R=1.3775 \mu \mathrm{m}$. The elemental composition of the glass plate is summarized in Table 3. Inspection of Fig. 7(A) reveals a significant dose increase shortly above the glass substrate only due to the presence of the substrate itself. Although this only affects an approximately $1 \mu \mathrm{m}$ thin layer of the tissue, it should however not be completely disregarded, especially when very thin tissue sections are being investigated, such as paraffinembedded sections cut in a microtome. For completeness, the inverse situation was also simulated, as shown in Fig. 7(B). In this case, the beam first traverses the glass plate and then the tissue sample. The dose profile is approximately symmetric to the previous case and once again a dose enhancement could be observed in the first $1 \mu \mathrm{m}$ section of the tissue.

Lastly, EGSnrc was used to simulate the dose profile within a tissue section using a setting that closely resembles the experimental configuration described in Section 3. For this purpose, a parallel X-ray beam with a photon energy $E=13.0 \mathrm{keV}$ and assuming a Gaussian beam profile with a FWHM of $1.4 \mu \mathrm{m}$ was simulated. To approximate the composition of the cardiac tissue used in the experiment described in Section 3, the ICRU material striated muscle (NIST standard material 202) was used with a mass density of $1.04 \mathrm{~g} \mathrm{~cm}^{-3}, \quad \mu / \rho=$ $3.1582 \mathrm{~cm}^{2} \mathrm{~g}^{-1}$ and $R=3.889 \mu \mathrm{m}$ and interpolated for $E=13.0 \mathrm{keV}$. The elemental composition of the muscle tissue matrix is shown in Table 4. From the above settings, the dose distribution
Figure 6

(A) Simulated pencil-beam dose kernels in a semi-infinite medium for different photon energies. Computation time for $10^{8}$ histories (i.e. $10^{8}$ incident photons and all subsequent events) is a few hours on a standard desktop PC. (B) Corresponding transversal dose profiles $D(r)$ in a depth $R<z \ll \mu^{-1}$ (evaluated at $z=10 \mu \mathrm{m}$ ). 


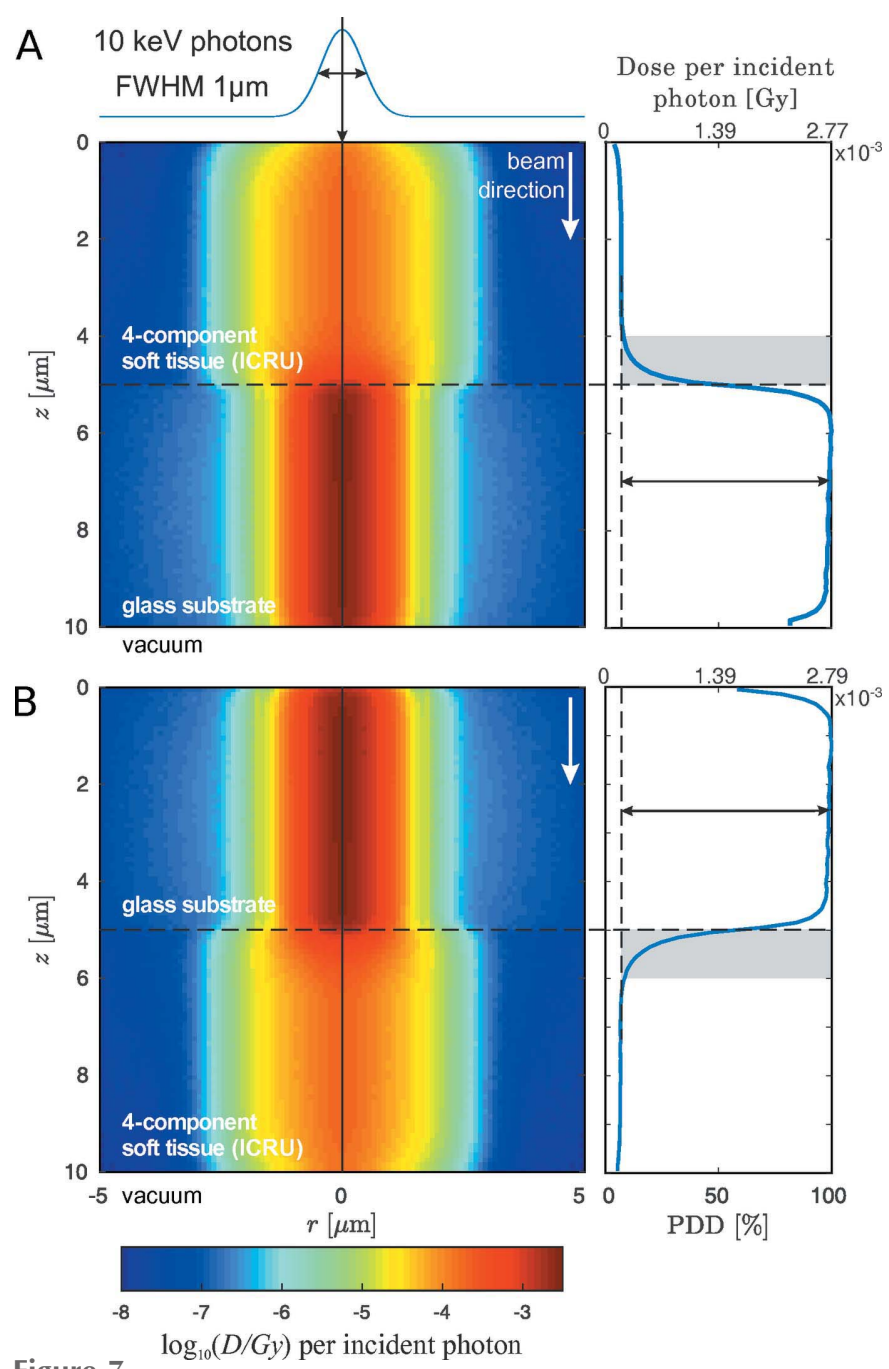

Figure 7

(A) MC simulation of a $10 \mathrm{keV}$ photon beam impinging onto a sample composed of a $5 \mu \mathrm{m}$ soft tissue film supported by a $5 \mu \mathrm{m}$ thin glass substrate. Close to the soft tissue/glass interface, the dose in the soft tissue film is considerably increased by back-scattered photoelectrons from the glass. One should be aware of this effect when very thin solid-supported soft tissue films are studied. The effect can be more clearly seen in the percentage depth dose (PDD) curve along the $z$-axis for the central line marked by a black line in the cylindrical $2 \mathrm{D}$ profile. The region of increased sample dose is highlighted in gray. (B) For completeness, the inverse situation of transmitting first through the glass substrate and then the soft tissue has also been simulated.

was simulated and is shown in Fig. 8(B). The radial dose profile at $z=10 \mu \mathrm{m}$ can be well approximated by a sum of two Gaussian components [see Ulmer \& Harder (1995); Gaussian 1 and Gaussian 2, compare Fig. 8], where the first Gaussian component corresponds to the illuminating photon field. The FWHM of the second component is $3.33 \mu \mathrm{m}$. It is noteworthy that the overall dose profile including its tails is hence around $5 \mu \mathrm{m}$, placing the previously assumed 'safety margins' for dose propagation in tissues on a solid footing. As an example, Nicolas et al. $(2017 b)$ used a $5 \mu \mathrm{m}$ step size for a beam with $2 \mu \mathrm{m} \times 3 \mu \mathrm{m}(\mathrm{FWHM})$. At the same time, the experiment described in Fig. 2 shows that a line step of $4-5 \mu \mathrm{m}$ (in the continuous scanning protocol) was also safe at the given parameters.
Table 4

Striated muscle.

\begin{tabular}{lcc}
\hline Element & $Z$ & $\begin{array}{c}\text { Mass fraction } \\
\text { in percent }\end{array}$ \\
\hline $\mathrm{H}$ & 1 & 10.20 \\
$\mathrm{C}$ & 6 & 12.30 \\
$\mathrm{~N}$ & 7 & 3.50 \\
$\mathrm{O}$ & 8 & 72.90 \\
$\mathrm{Na}$ & 11 & 0.080 \\
$\mathrm{Mg}$ & 12 & 0.020 \\
$\mathrm{P}$ & 15 & 0.20 \\
$\mathrm{~S}$ & 16 & 0.50 \\
$\mathrm{~K}$ & 19 & 0.30 \\
\hline
\end{tabular}

\section{Summary and conclusion}

We have identified a parameter window of dose and spatial dose distribution under which the SAXS signal, more specifically the equatorial reflections from hydrated cardiac tissue slices, is unaffected by radiation damage, for sufficiently short acquisition time and large enough distance between successive exposures. Conversely, tighter focusing and smaller steps, for example towards submicrometre spatial resolution, are not feasible without either losing the signal (at too small a dose) or affecting the signal due to radiation damage. These conclusions have been corroborated by MC simulations carried out with the software package EGSnrc, which we use here to simulate dose profiles for microfocus X-ray beam experiments on thin soft tissue sections. The results provide additional evidence for the inclusion of a 'safety margin' in the step sizes in scanning experiments with microfocused beams. Since dose is deposited via secondary electrons, the photoelectron range $R$ at the relevant energies has to be considered instead of the beam size to obtain a good estimate for the area that will be affected by radiation damage. We would also like to point out that the usefulness of MC simulations using EGSnrc is not limited to X-ray diffraction experiments but could easily be extended to coherent imaging, including also more complex sample and source geometries. It is therefore another helpful addition to the set of dose simulation software packages (Drouin et al., 2007; Bury et al., 2018) available to the synchrotron research community.

The above analysis is important for two reasons. (i) Microfocused and nanofocused X-ray beams as used in scanning diffraction and coherent diffractive imaging can easily introduce dose of the order of several MGy and even GGy to the sample, which makes it an important goal to optimize the data collection strategy in a similar fashion as is being discussed in X-ray crystallography (Zeldin et al., 2013; Owen et al., 2014; Gao et al., 2018). (ii) The results give further evidence that soft tissue samples studied at RT require relaxed focusing and an optimized scanning scheme. In many cases, such as for experiments on living cells, only the selected probing of isolated pristine spots in the sample appears reasonable. Since biological systems are very sensitive to temperature changes, future work should also explore in greater detail how sample heating relates to absorbed dose in the sample. In addition, future extensions of this work could 


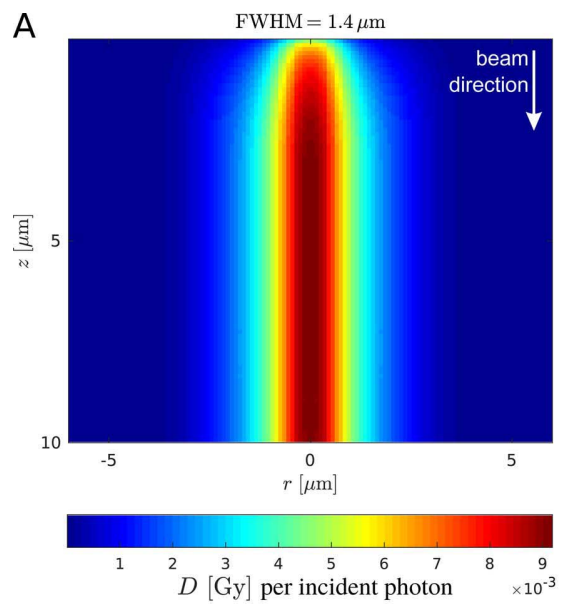

Figure 8

(A) Simulated dose profile in a $10 \mu \mathrm{m}$ muscle tissue medium [NIST material striated muscle (ICRU)] for a photon energy of $13 \mathrm{keV}$ and a beam size (FWHM) of $1.40 \mu \mathrm{m}$. (B) Corresponding transversal dose profiles $D(r)$ in a depth $R<z \ll \mu^{-1}$ (evaluated at $z=10 \mu \mathrm{m}$ ). The transversal dose profile can be separated into two Gaussian components where one component (Gaussian 1) corresponds to the beam profile and the second component (Gaussian 2) to the broadened dose profile. The width (FWHM) of the second Gaussian is $3.33 \mu \mathrm{m}$.

include cryogenic protection and/or the use of radical scavengers.

\section{APPENDIX $A$}

\section{EGSnrc simulation}

A compressed file containing the parameter settings of EGSnrc for the example shown in Fig. 4, as well as a material definition file and a file containing the expected output for the pre-defined simulation, is included as supporting information for this manuscript. In addition, a Matlab-file was added to simplify visualization. EGSnrc was installed on a standard desktop computer (Dell Optiplex 7020) with a Fedora 27 operating system. The EGSnrc software was downloaded as a rip file (EGSnrc-master.zip). The software was then installed using the executable EGSnrc-configure - linux. Both files are available online at https://github.com/nrc-cnrc/EGSnrc/releases/tag/ v2018. For general information, installation and usage of EGSnrc, the reader is referred to the extensive EGSnrc manual available from the following web address: http://nrccnrc.github.io/EGSnrc/.

In the simulations presented here, the kinetic energy of an electron below which it deposits its entire energy in the volume element and is considered 'absorbed' by the medium was set to $1 \mathrm{keV}$. A second important parameter is the maximum energy loss per step (between two interactions). We have set this parameter to its recommended value of 0.25 (Kawrakow, 2000).

\section{Acknowledgements}

We thank Manfred Burghammer for valuable discussions related to the effect of radiation damage in spatially resolved SAXS experiments. Assistance during beam time by Marius Reichardt and Marten Bernhardt is gratefully acknowledged.
Bernhardt, M., Priebe, M., Osterhoff, M., Wollnik, C., Diaz, A., Salditt, T. \& Rehfeldt, F. (2016). Biophys. J. 110, 680-690.

Bukreeva, I., Fratini, M., Campi, G., Pelliccia, D., Spanò, R., Tromba, G., Brun, F., Burghammer, M., Grilli, M., Cancedda, R., Cedola, A. \& Mastrogiacomo, M. (2015). Front. Bioeng. Biotechnol. 3, 133.

Bunk, O., Bech, M., Jensen, T. H., Feidenhans'l, R., Binderup, T., Menzel, A. \& Pfeiffer, F. (2009). New J. Phys. 11, 123016.

Bury, C. S., Brooks-Bartlett, J. C., Walsh, S. P. \& Garman, E. F. (2018). Protein Sci. 27, 217-228.

Cowan, J. A. \& Nave, C. (2008). J. Synchrotron Rad. 15, 458-462.

Deyhle, H., Bunk, O. \& Müller, B. (2011). Nanomedicine: Nanotechnol. Biol. Med. 7, 694-701.

Deyhle, H., White, S. N., Bunk, O., Beckmann, F. \& Müller, B. (2014). Acta Biomater. 10, 355-364.

Dickerson, J. L. \& Garman, E. F. (2019). J. Synchrotron Rad. 26, $922-$ 930.

Drouin, D., Couture, A. R., Joly, D., Tastet, X., Aimez, V. \& Gauvin, R. (2007). Scanning, 29, 92-101.

Faulkner, H. M. L. \& Rodenburg, J. M. (2004). Phys. Rev. Lett. 93, 023903.

Gao, Y., Xu, W., Shi, W., Soares, A., Jakoncic, J., Myers, S., Martins, B., Skinner, J., Liu, Q., Bernstein, H., McSweeney, S., Nazaretski, E. \& Fuchs, M. R. (2018). J. Synchrotron Rad. 25, 1362-1370.

Garman, E. F. \& Weik, M. (2017). J. Synchrotron Rad. 24, 1-6.

Giannini, C., Ladisa, M., Lutz-Bueno, V., Terzi, A., Ramella, M., Fusaro, L., Altamura, D., Siliqi, D., Sibillano, T., Diaz, A., Boccafoschi, F. \& Bunk, O. (2019). IUCrJ, 6, 267-276.

Giannini, C., Siliqi, D., Ladisa, M., Altamura, D., Diaz, A., Beraudi, A., Sibillano, T., De Caro, L., Stea, S., Baruffaldi, F. \& Bunk, O. (2014). J. Appl. Cryst. 47, 110-117.

Gourrier, A., Li, C., Siegel, S., Paris, O., Roschger, P., Klaushofer, K. \& Fratzl, P. (2010). J. Appl. Cryst. 43, 1385-1392.

Granke, M., Gourrier, A., Rupin, F., Raum, K., Peyrin, F., Burghammer, M., Saïed, A. \& Laugier, P. (2013). PLoS One, 8, e58043.

Hall, E. J. \& Giaccia, A. J. (2012). Radiobiology for the Radiologist, 7th ed. Philadelphia: Lippincott Williams \& Wilkins.

Hémonnot, C. Y. J. \& Köster, S. (2017). ACS Nano, 11, 8542-8559.

Henderson, R. (1990). Proc. R. Soc. Lond. B Biol. Sci. 241, 6-8.

Howells, M. R., Beetz, T., Chapman, H. N., Cui, C., Holton, J. M., Jacobsen, C. J., Kirz, J., Lima, E., Marchesini, S., Miao, H., Sayre, D., Shapiro, D. A., Spence, J. C. H. \& Starodub, D. (2009). J. Electron Spectrosc. Relat. Phenom. 170, 4-12. 
Karunaratne, A., Davis, G. R., Hiller, J., Esapa, C. T., Terrill, N. J., Brown, S. D. M., Cox, R. D., Thakker, R. V. \& Gupta, H. S. (2012). Bone, 51, 553-562.

Kawrakow, I. (2000). Med. Phys. 27, 485-498.

Leroy, C. \& Rancoita, P.-G. (2013). Principles of Radiation Interaction in Matter and Detection, 3rd ed. Singapore: World Scientific.

Lichtenegger, H., Müller, M., Paris, O., Riekel, C. \& Fratzl, P. (1999). J. Appl. Cryst. 32, 1127-1133.

McDermott, P. N. (2016). Tutorials in Radiotherapy Physics: Advanced Topics with Problems and Solutions, 1st ed. CRC Press.

Märten, A., Fratzl, P., Paris, O. \& Zaslansky, P. (2010). Biomaterials, 31, 5479-5490.

Matsubara, I. (1980). Annu. Rev. Biophys. Bioeng. 9, 81-105.

Müller, B., Deyhle, H., Bradley, D. A., Farquharson, M., Schulz, G., Müller-Gerbl, M. \& Bunk, O. (2010). Eur. J. Nanomed. 3, 30-33.

Müller, M. (2009). Mater. Sci. Forum, 599, 107-125.

Nave, C. \& Hill, M. A. (2005). J. Synchrotron Rad. 12, 299-303.

Nicolas, J.-D., Bernhardt, M., Krenkel, M., Richter, C., Luther, S. \& Salditt, T. (2017b). J. Appl. Cryst. 50, 612-620.

Nicolas, J.-D., Bernhardt, M., Markus, A., Alves, F., Burghammer, M. \& Salditt, T. (2017a). J. Synchrotron Rad. 24, 1163-1172.

Nicolas, J.-D., Bernhardt, M., Schlick, S. F., Tiburcy, M., Zimmermann, W.-H., Khan, A., Markus, A., Alves, F., Toischer, K. \& Salditt, T. (2019). Prog. Biophys. Mol. Biol. 144, 151-165.

Owen, R. L., Paterson, N., Axford, D., Aishima, J., Schulze-Briese, C., Ren, J., Fry, E. E., Stuart, D. I. \& Evans, G. (2014). Acta Cryst. D70, 1248-1256.

Riekel, C., Burghammer, M., Davies, R. J., Di Cola, E., König, C., Lemke, H. T., Putaux, J.-L. \& Schöder, S. (2010). J. Synchrotron Rad. 17, 743-750.
Riley, P. A. (1994). Int. J. Radiat. Biol. 65, 27-33.

Rinnerthaler, S., Roschger, P., Jakob, H. F., Nader, A., Klaushofer, K. \& Fratzl, P. (1999). Calcif. Tissue Int. 64, 422-429.

Sakdinawat, A. \& Attwood, D. (2010). Nat. Photon. 4, 840-848.

Sanishvili, R., Yoder, D. W., Pothineni, S. B., Rosenbaum, G., Xu, S., Vogt, S., Stepanov, S., Makarov, O. A., Corcoran, S., Benn, R., Nagarajan, V., Smith, J. L. \& Fischetti, R. F. (2011). Proc. Natl Acad. Sci. USA, 108, 6127-6132.

Seco, J. \& Verhaegen, F. (2013). Monte Carlo Techniques in Radiation Therapy, 1st ed. CRC Press.

Stangl, J., Mocuta, C., Chamard, V. \& Carbone, D. (2014). Nanobeam $X$-ray Scattering: Probing Matter at the Nanoscale. Wiley-VCH.

Storm, S., Ogurreck, M., Laipple, D., Krywka, C., Burghammer, M., Di Cola, E. \& Müller, M. (2015). J. Synchrotron Rad. 22, 267-272.

Tesch, W., Eidelman, N., Roschger, P., Goldenberg, F., Klaushofer, K. \& Fratzl, P. (2001). Calcif. Tissue Int. 69, 147-157.

Thibault, P., Dierolf, M., Menzel, A., Bunk, O., David, C. \& Pfeiffer, F. (2008). Science, 321, 379-382.

Ulmer, W. \& Harder, D. (1995). Z. Med. Phys. 5, 25-30.

Wagermaier, W., Gupta, H. S., Gourrier, A., Paris, O., Roschger, P., Burghammer, M., Riekel, C. \& Fratzl, P. (2007). J. Appl. Cryst. 40, 115-120.

Weinhausen, B., Nolting, J.-F., Olendrowitz, C., Langfahl-Klabes, J., Reynolds, M., Salditt, T. \& Köster, S. (2012). New J. Phys. 14, 085013.

Weinhausen, B., Saldanha, O., Wilke, R. N., Dammann, C., Priebe, M., Burghammer, M., Sprung, M. \& Köster, S. (2014). Phys. Rev. Lett. 112, 088102 .

Zeldin, O. B., Gerstel, M. \& Garman, E. F. (2013). J. Synchrotron Rad. 20, 49-57. 